\title{
Intelligent automatic overtaking system using vision for vehicle detection
}

\author{
Vicente Milanés \\ David F. Llorca \\ Jorge Villagrá \\ Joshué Pérez Carlos Fernández \\ Ignacio Parra \\ Carlos González Miguel A. Sotelo
}

\begin{abstract}
A B S T R A C T
There is clear evidence that investment in intelligent transportation system technologies brings major social and economic benefits. Technological advances in the area of automatic systems in particular are becoming vital for the reduction of road deaths. We here describe our approach to automation of one the riskiest autonomous manœuvres involving vehicles - overtaking. The approach is based on a stereo vision system responsible for detecting any preceding vehicle and triggering the autonomous overtaking manœuvre. To this end, a fuzzy-logic based controller was developed to emulate how humans overtake. Its input is information from the vision system and from a positioning-based system consisting of a differential global positioning system (DGPS) and an inertial measurement unit (IMU). Its output is the generation of action on the vehicle's actuators, i.e., the steering wheel and throttle and brake pedals. The system has been incorporated into a commercial Citroën car and tested on the private driving circuit at the facilities of our research center, CAR, with different preceding vehicles - a motorbike, car, and truck - with encouraging results.
\end{abstract}

\section{Introduction}

The development of advanced driver assistance systems (ADAS) to aid in driving-related tasks has a key role to play in the automotive field (Lu, Wevers, van der Heijden, \& Heijer, 2004). Assistance systems - to prevent accidents or to make driving safer - which alert the driver of imminent risks through visual or audible signals have been included in commercial cars in recent years (Castro, Delgado, \& Medina, 2011; Chang, Tsai, \& Young, 2010; Geronimo, Lopez, Sappa, \& Graf, 2010; Lindgren, Angelelli, Mendoza, \& Chen, 2009; Perez et al., 2010). Although these techniques are necessary and provide a warning to the driver, their dependence on human reaction time for a decision to be made remains a problem.

The first system introduced in commercial vehicles with the potential to influence traffic safety was adaptive cruise control (ACC) (Kestinga, Treibera, Schönhofa, \& Helbing, 2008; Peng, 2010). This was an extension of cruise control (CC) - CC allows the driver to set a driving speed - in which the vehicle is capable of following a leading car on highways by automatic action on the throttle and brake pedals, i.e., longitudinal control. Lateral or steering control, however, remains one of the toughest challenges in the development of commercial ADAS. The currently most advanced system is lane keeping assistance (LKA) (Wang, Lin, \& Chen, 2010; Wang et al., 2005; Wu et al., 2008) which provides limited additional steering torque to aid the driver in maintaining the vehicle within the lane if a potential lane departure is detected. Complete lateral vehicle control has yet to be solved by the automotive sector.

Research on developing automated vehicles to improve highway safety and efficiency is one of the most extensively studied topics in the field of intelligent transportation systems. The first relevant results with respect to steering control were obtained in the 1990s. The Vislab (Broggi, Bertozzi, Fascioli, Lo, \& Piazzi, 1999) and Navlab (Pomerleau, 1995) research groups in Italy and the United States, respectively, use video images to locate the vehicle's location on the road. In longitudinal control, California Partners for Advanced Transit and Highways (PATH) (Godbole \& Lygeros, 1993) developed a control system in which cars moved along the highway in tightly spaced platoons.

Of all manœuvres, overtaking is one of the most complex since it combines both lateral and longitudinal control. The work to be described here is part of a collaboration between the AUTOPIA program of the Centre for Automation and Robotics (CAR, CSIC-UPM) and the RobeSafe group of Alcala University. Its aim is to develop a system capable of performing overtaking autonomously using as input data from a vision system to acquire information about vehicles in the vicinity, and an inertial measurement unit (IMU) with a differential global positioning system (DGPS) to obtain the 
vehicle's positioning. A fuzzy-logic based controller is responsible for sending the appropriate output to the vehicle's actuators the steering wheel, and throttle and brake pedals.

The problem of automating the overtaking manœuvre has been tackled from different points of view. A simulated guidance-based on-line trajectory planning algorithm - based on the principles of Rendezvous Guidance - is defined in Usman and Kunwar (2009). A mathematical model is used in Shamir (2004) to design a smooth and comfortable optimal lane-change trajectory for simulation purposes.

Different methods for vehicle detection in overtaking have been proposed. In Zhu, Comaniciu, Pellkofer, and Koehler (2006) a fusion of dynamic modeling and robust information is used for motion estimation. In Wang, Bebis, and Miller (2005), detection is performed by modeling the background of a traffic scene divided into dynamic and quasi-static regions. A conflict-probability-estimation-based overtaking control method is presented in Wang, Yang, and Yang (2009) using a four-wheeled electric vehicle denominated a cybercar. The same kind of vehicle is used in Feng, Rongben, and Ronghui (2008) in which a vision navigation system is used to perform overtaking manœuvres.

In previous work (Naranjo, Gonzalez, Garcia, \& de Pedro, 2008), a lane-change controller was developed in the AUTOPIA program, determining experimentally the relationship between the speed and the lane-change distance using vehicle-to-vehicle (V2V) communication to perform the manœuvre. This system was based on a priori knowledge of the state of the road. However, the development of ADAS in mass-produced cars allows one to assume that autonomous or semi-autonomous vehicles will be driving on our roads in the medium to long term. Consequently, it is obligatory to have a system capable of permitting the coexistence of these kinds of vehicle with today's human driven vehicles on the roads. Given this premise, all the sensorial information will have to be on board the autonomous vehicle, and the path-tracking actually generated will have to depend on the traffic conditions. Our goal is to develop a system whose behavior is as close to that of the human driver as we can make it. To this end, different trajectories will be generated depending on the leading vehicle's characteristics as obtained from vision-based information.

The rest of the article is structured as follows. Section 2 describes the manœuvre to be implemented. Section 3 gives a brief description of the mass-produced car used in the experimental phase. Section 4 presents the vision-based traffic information system developed to activate the autonomous control system, which is detailed in Section 5 for both longitudinal and lateral control. Section 6 includes the experimental results with the prototype autonomous vehicle on CAR's private driving circuit. Finally, Section 7 presents the conclusions and describes the next steps in our research.

\section{Description of the problem}

An overtaking manœuvre is a driving action involving a rapid movement to negotiate an obstacle. This movement includes a steering wheel turn combined with a speed increase. The former is applied to perform a double change of lane - first to overtake the obstacle, and then to return to the original lane in a progressive steering change. Our goal is to develop a system capable of distinguishing between the vehicles that may be on the road - truck, car, or motorbike - and of performing the automatic manœuvre as human drivers do.

Let us consider the general case in which a vehicle is driving in the right lane along a straight stretch of a highway. One can distinguish three kinds of preceding vehicles according to their length and width. To make overtaking close to that done by humans, dif- ferent path references were set to perform the manœuvre with safety. Fig. 1 presents the vehicles to be overtaken and the possible trajectories. As can be seen, the wider the preceding vehicle is, the greater the lateral movement, and the longer the preceding vehicle, the more prolonged the manœuvre.

An automatic overtaking manœuvre can be naturally divided into two stages. The first is to detect when the manœuvre can be carried out. To this end, a vision-based system was developed to analyze the conditions of the environment, permanently checking whether it is possible to overtake so as to activate the automatic manœuvre.

The second stage consists of performing the manœuvre autonomously. One has to consider different lateral displacements according to the nature of the preceding vehicle. The time of greatest risk is when the overtaker and the overtaken are running in parallel. The greater the speed, the less time is spent in the overtaking lane. Finally, the vehicle has to return to the original lane. Since safety is the priority parameter in this kind of cooperative manœuvre, time constraints must be imposed for the manœuvre to be performed autonomously. With these premises, the following variables involving the preceding vehicle are taken into account (see Fig. 1):

Time-to-collision (TTC) obtained by dividing the distance between the preceding vehicle and the automatic one by their relative speed. These two parameters are obtained from the vision system.

Preceding vehicle width $\left(P V_{W}\right)$ obtained from the vision system. It is used to determine how fast the steering change will have to be.

Preceding vehicle length $\left(P V_{L}\right)$ estimated from the vision system. It determines the time the automatic vehicle will spend in the overtaking lane - how sudden the speed change has to be.

The rest of the variables needed to carry out the autonomous manœuvre are obtained from the vehicle's on-board sensor systems, the principal variable being the position which is given by a differential global positioning system (DGPS) combined with an inertial measurement unit (IMU) (Milanés, Naranjo, Gonzalez, Alonso, \& de Pedro, 2008).

Errors inherent to the use of the sensors (cameras, GPS receivers, and the IMU) may lead to some imprecision in the measurements. For this reason, fuzzy logic was selected as control technique since it is a well-tested method for dealing with this kind of system, provides good results, and can incorporate human procedural knowledge into the control algorithms. It also allows the designer to partially mimic human driving behavior.

\section{Automated prototype vehicle}

A commercial convertible Citroën C3 Pluriel equipped with automatic driving capabilities was used in the real experiments. This section presents a brief description of the vehicle's equipment.

To automate a vehicle, the actuators involved in the drivingrelated tasks have to be modified. To this end, a power stage was developed in parallel with the vehicle's electronic control unit. This power stage was pulse-width modulation (PWM) controlled (Naranjo, Gonzalez, Garcia, de Pedro, \& Haber, 2005). A switch permits either the original steering system or the automatic one to be selected. For the throttle, an analogue signal replaces the action on the throttle when a switch is on. Finally, an electro-hydraulic braking system was installed in the vehicle's boot to manage the vehicle's brakes (Milanés, González, Naranjo, Onieva, \& De Pedro, 2010). 


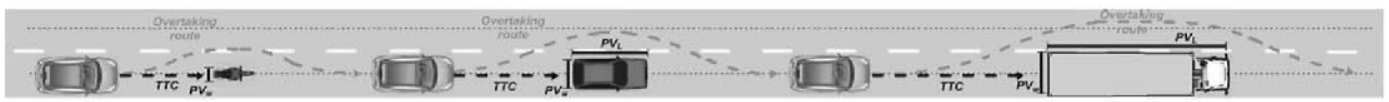

Fig. 1. Representation of cases of overtaking.

Sensors were installed to receive information on the vehicle's environment and for the vehicle's positioning. The former is obtained via a vision-based system (Llorca et al., 2009; Parra et al., 2007), and the latter uses a fusion GPS/IMU system (Milanés et al., 2008). Fig. 2 shows the prototype vehicle used in the experimental phase.

\section{Vision-based vehicle detection system}

The vision system installed in the prototype vehicle is responsible for detecting any preceding vehicle - i.e., motorbike, car, or truck - and determining its width and length, and its distance away.

\subsection{Description of the system}

The commonest approach to vehicle detection has until now been to use active acoustic-based (Chellappa, Qian, \& Zheng, 2004), radar-based (Park, Kim, Kang, \& Koo, 2003), or laser-based (Hancock et al., 1998; Wang, Thorpe, \& Suppe, 2003) sensors. However, passive sensors, in particular optical sensors, have been attracting much of the attention of the research community and the industry because of their two main characteristics: cheapness and potential new applications such as lane departure warning (LDW), pedestrian detection, traffic sign recognition, etc. Of all these applications, algorithms for detecting and tracking vehicles (Mandellos, Keramitsoglou, \& Kiranoudis, 2011) are one of the most important topics in the field. A review of the background concerning vehicle detection, covering both active and passive sensors, may be found in Sun, Bebis, and Miller (2006).

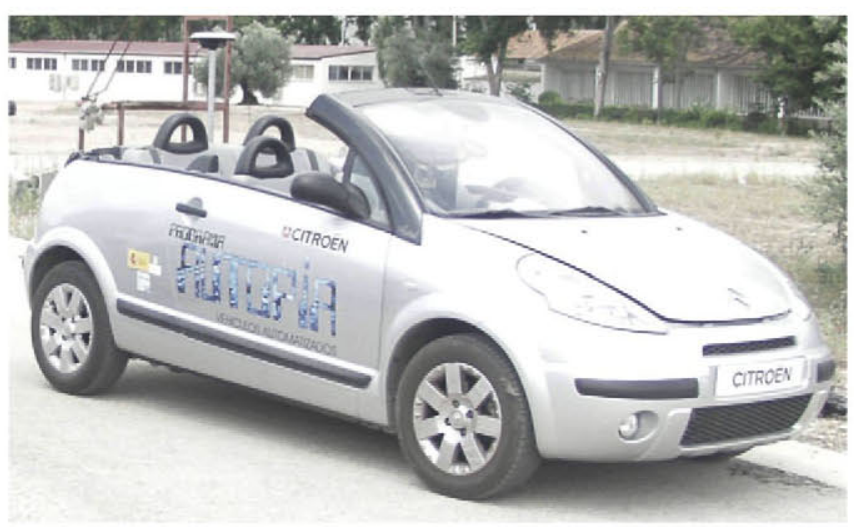

Fig. 2. Automated prototype vehicle equipped with DGPS, IMU, and vision system.
Fig. 3 is an overview of the proposed vision-based vehicle detection system. Initial regions of interest (ROIs) are computed using the monocular method described in Llorca, Sánchez, Ocaña, and Sotelo (2010). Lane markings are detected, thereby reducing the vehicle search area. If no lane markings are detected, a basic ROI corresponding to a straight road is used instead. Vehicle candidate regions are selected using a combination of symmetries (vertical edges, horizontal edges, and gray level symmetries) and white hat and Canny features together with a non-maximum suppression procedure which removes overlapped candidates (Llorca \& Sánchez et al., 2010).

A second camera was added to obtain a more detailed description of the leading vehicle's features. Stereo processing results in a dense disparity map which allows the 3D position, the TTC, the width, and the length of the vehicle to be estimated accurately. The camera pitch angle is estimated dynamically by means of the so-called virtual disparity map from which regions corresponding to the ground-plane can easily be removed (Llorca et al., 2009). One thus obtains a more precise specification of the areas of the ground where vehicles are to be expected (see Fig. 4).

Each candidate vehicle region output by the monocular module is verified in stereo mode by counting the number of depth features corresponding to the filtered dense disparity map. In particular, the locations where the number of depth features exceeds a certain fraction of the window area are passed onto subsequent modules, thus ensuring that each candidate region corresponds to a real 3D object.

Depth features are also used to obtain geometrical information about the candidate vehicle. First, the 3D position of each candidate is found by applying the so-called 3D subtractive clustering algorithm (Parra et al., 2007) to the set of 3D points contained in the ROI. Consecutive 3D measurements, after filtering, are used to compute the relative velocity $(\mathrm{Vr})$ and the host-to-vehicle (H2V) TTC. The leading vehicle's speed is then simply the sum of the host's speed taken from the CAN bus and the relative velocity. The absolute and relative depth estimation errors are determined by the stereo quantization error procedure as proposed in Llorca, Sotelo, Parra, Ocaña, and Bergasa (2010).

The preceding vehicle's width, used to optimize the lateral offset of the overtaking trajectory, is computed as follows. The verified $\mathrm{ROI}$ is enlarged by $10 \%$, and the value of the mode of the depth features contained in the ROI is calculated. For each column of the enlarged ROI, the number of depth features whose values are equal to the mode plus-or-minus a certain tolerance is determined, generating a histogram like the one depicted in Fig. 5. The lateral boundaries of the histogram are then found, and the vehicle's width in pixels is computed. The 3D vehicle's width is finally obtained using this estimated width in pixels, the depth of the

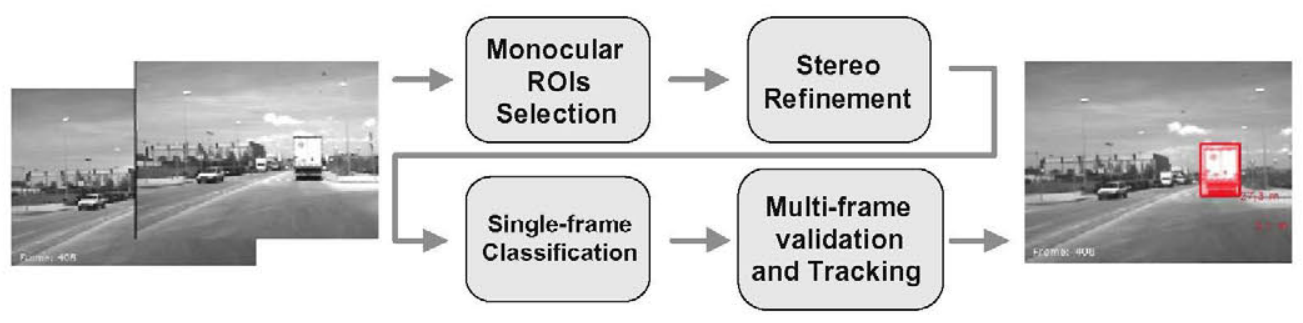

Fig. 3. The stages of vehicle detection. 


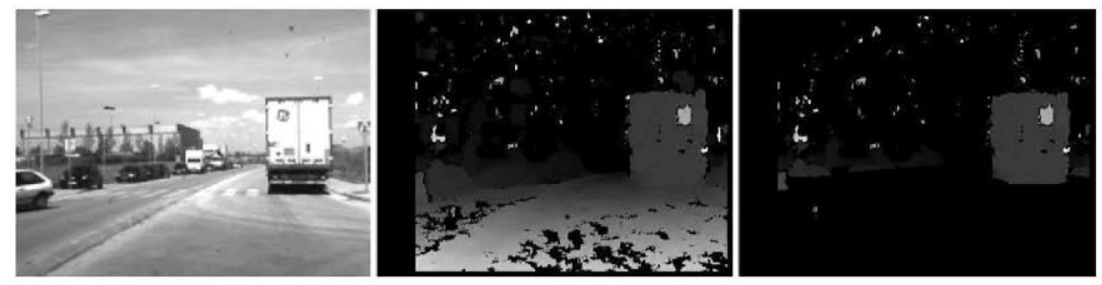

Fig. 4. From left to right: original image, dense disparity map, and filtered map (without ground-plane points or points that are very high).
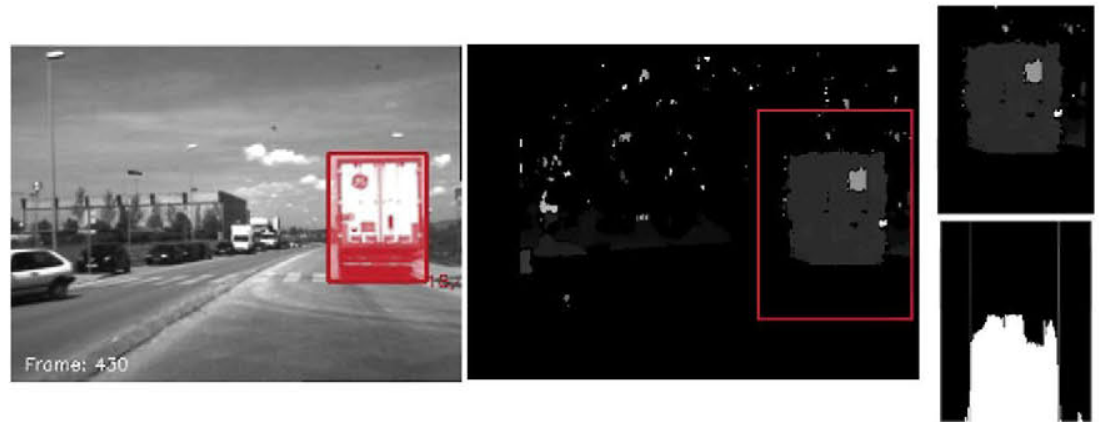

Fig. 5. From left to right: ROI in the original image; enlarged ROI in the filtered dense disparity map; column histogram and lateral boundaries.
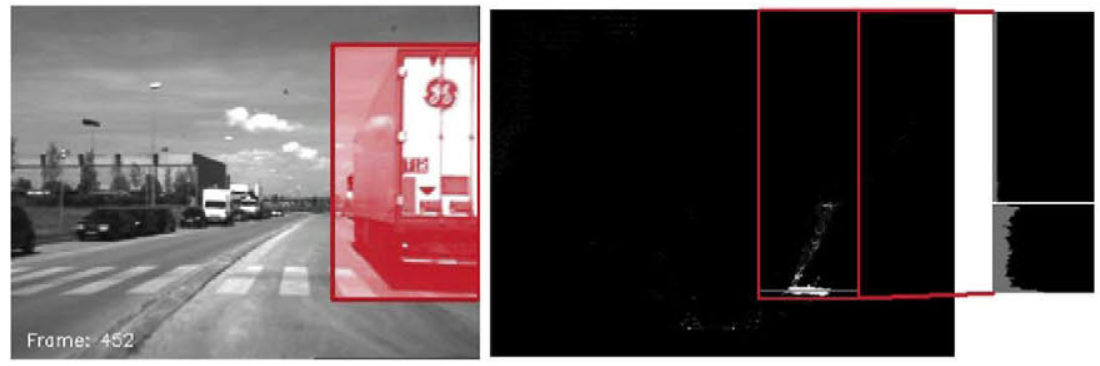

Fig. 6. From left to right: ROI in the original image; $\mathrm{XOZ}$ map and the ROI used to compute the length; row histogram and lower bound of the dark area.

vehicle, prior knowledge of the camera geometry, and the flatworld assumption (acceptable in most cases).

The leading vehicle's length is an important parameter that can be used to minimize the time the host vehicle remains in the overtaking lane. This length can not be computed from the rear view of the leading vehicle. Once the host vehicle has entered the overtaking lane, however, the side of the leading vehicle is visible to the stereo pair (see Fig. 6). To estimate the leading vehicle's length in that position, we use the XOZ map (bird's eye view) as is depicted in Fig. 6 in which the leading vehicle appears with an L-shape. A histogram is formed from the counts of points of each row corresponding to an ROI located from the rear part of the vehicle to the top of the XOZ map. This histogram is analyzed to detect the lower bound of the region in which no points are detected. Subtracting this lower bound of the dark area from the rear part of the vehicle in the $\mathrm{XOZ}$ map gives the vehicle's length directly.

The selected and verified candidates are classified by means of a linear support vector machine (SVM) classifier in combination with features of the "histograms of oriented gradients" technique (Llorca \& Sánchez et al., 2010). A predefined number (empirically set to 3 ) of consecutive identifications of an object classified as a vehicle triggers the data association and tracking stages. The data association problem is addressed by using feature matching techniques. Harris features are detected and matched between two consecutive frames, as in Llorca and Sánchez et al. (2010). Tracking is implemented using a Kalman filter with a constant velocity model. The state of the filter is modeled as $S=[x, z, w, l, \dot{x}, \dot{z}]^{T}$ with $x / z$ being the $\mathrm{H} 2 \mathrm{~V}$ lateral/longitudinal position, $w / l$ the width/ length of the leading vehicle, and $\dot{x} / \dot{z}$ the $\mathrm{H} 2 \mathrm{~V}$ relative velocity in the world. Note that length measurements are only valid once the side of the leading vehicle has become visible.

\subsection{System validation}

The vehicle detection system runs in real time $(25 \mathrm{~Hz})$ with an image resolution of $320 \times 240$ pixels, a baseline of $30 \mathrm{~cm}$, and a camera focal length of $8 \mathrm{~mm}$. In a first experiment, we evaluated the different parameters provided by the vision sensor, i.e., the $\mathrm{H} 2 \mathrm{~V}$ distance, the relative speed, the TTC, and the leading vehicle's width and length, together with their corresponding errors. A set of sequences was recorded in which a driver was requested to perform an overtaking manœuvre in a two-lane road at approximately $25 \mathrm{~km} / \mathrm{h}$. Three types of vehicle of different sizes (a motorbike, a car, and a truck; see Fig. 7) were parked in the right-hand (nonovertaking) lane, so that the host vehicle overtakes these vehicles at a relative speed of around $25 \mathrm{~km} / \mathrm{h}$. Besides the stereo vision sensor, two DGPSs are used, one placed at the lead vehicle's position and the other on board the host vehicle (see Fig. 8). The measurements supplied by these DGPSs (after linear interpolation due to their low sampling frequency $-5 \mathrm{~Hz}$ ) are taken to be the ground truth. 

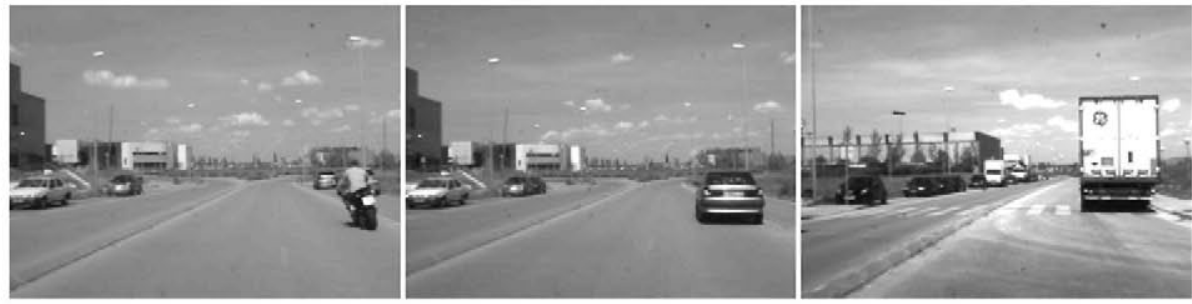

Fig. 7. The three vehicles used in the experiments.

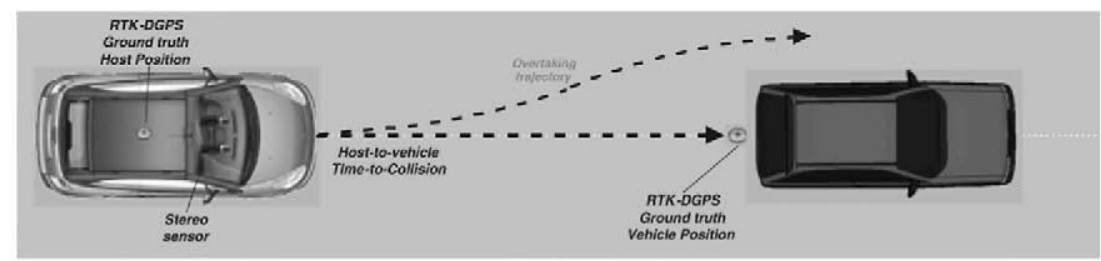

Fig. 8. Scheme of the manual driving experiment.

Fig. 9 plots the stereo and the DGPS depth estimates, including the absolute error and the stereo depth estimation uncertainties (Llorca et al., 2010), of the experiment in which the car is the vehicle overtaken. The DGPS H2V, which is taken to be the ground truth, lies mostly within the limits of the stereo depth estimate including their corresponding uncertainties. As can be observed, the greater the depth the greater the absolute error. Depth estimates may not be reliable at long distances (absolute errors). However, the absolute error decreases as the H2V distance decreases.

Although the depth estimation errors are sufficiently small for the precision required in the present application, those of the discrete estimate of the relative speed from the Kalman state variable $\dot{z}$ are not. As demonstrated in Stein, Mano, and Shashua (2003), the discrete difference between two noisy depth estimation values introduces a considerable error in the relative speed computation for non-infinitesimal $\Delta t$, and this clearly limits the vision system's precision. Those authors propose an optimal value for $\Delta t$ which is directly proportional to depth. In the present case, we define a practical value of $\Delta t=1 \mathrm{~s}$ and compute the average speed of the last 25 frames. This approach is very effective in practice. Fig. 10 depicts the relative speed obtained from the CAN bus (recall that the leading vehicle is parked in this experiment), that computed by means of DGPS H2V distance values, the discrete relative speed provided by the Kalman filter, and the relative speed computed at each second. One observes that the discrete values of the stereo relative speed are not at all useful. However, the proposed approach described above provides relative speed estimates that are accurate enough for the application's requirements - the root-mean-squared-error (RMSE) is around $3 \mathrm{~km} / \mathrm{h}$.

The above method for estimating the relative speed allows the H2V TTC to be computed with reasonable accuracy. Fig. 11 depicts the TTC estimated by means of DGPS measurements (ground truth) and by the stereo vision system. The absolute error is less than $0.3 \mathrm{~s}$ for a TTC below $6 \mathrm{~s}$.

The results of estimating the leading vehicle's width and length are depicted in Fig. 12. One observes that the width of the car estimated by the stereo vision system only achieves accuracy from frame 505, i.e., from distances of less than $25 \mathrm{~m}$. The width estimate degenerates from frame number 540 due to the lane change process during which the car is not fully visible. The length estimation procedure is only triggered once the host vehicle is mostly located in the overtaking lane, when the side of the car ahead has become visible.

Further details of the results of the three experiments (motorbike, car, and truck) are given in Tables $1-5$. One observes that the relative speed, depth, TTC, and width estimates are not accurate at long distances due to the stereo quantization error. However, as the $\mathrm{H} 2 \mathrm{~V}$ distance decreases all these estimates gain in precision. The range for motorbikes is $10 \mathrm{~m}$ less than for cars and trucks. The relative speed is computed with a RMSE of around

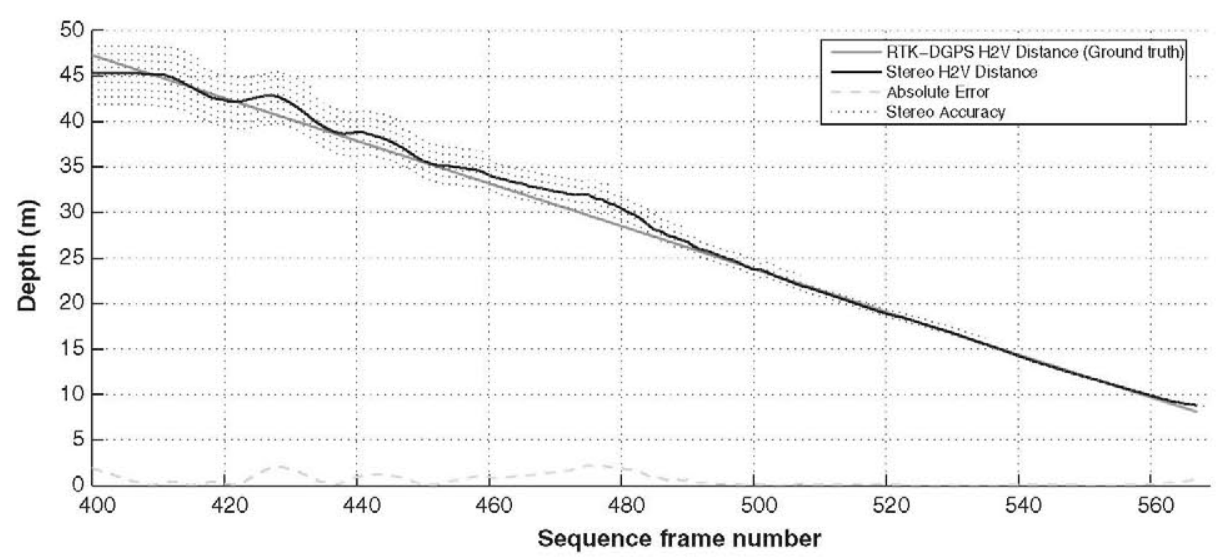

Fig. 9. Stereo and DGPS depth including absolute and depth estimation errors in the experiment in which the car is overtaken. 


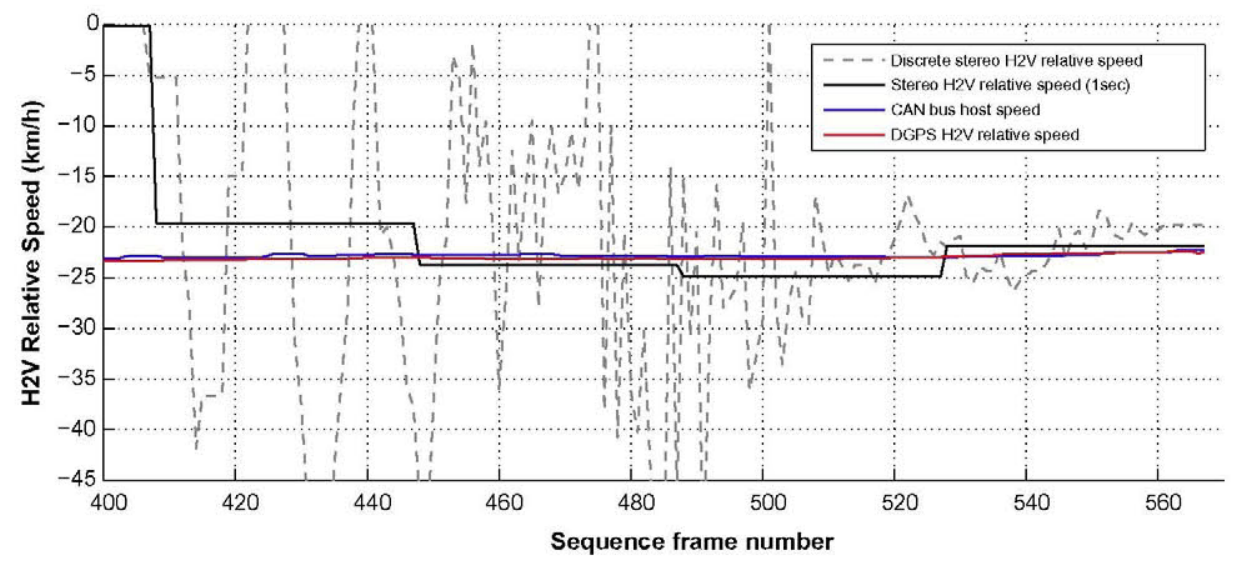

Fig. 10. $\mathrm{H} 2 \mathrm{~V}$ relative speed $(\mathrm{km} / \mathrm{h})$ taken from the CAN bus (the leading vehicle is parked), and from the DGPS depth values and the discrete stereo measurements, and estimated by averaging the discrete stereo values at each second, in the experiment where the car is overtaken.

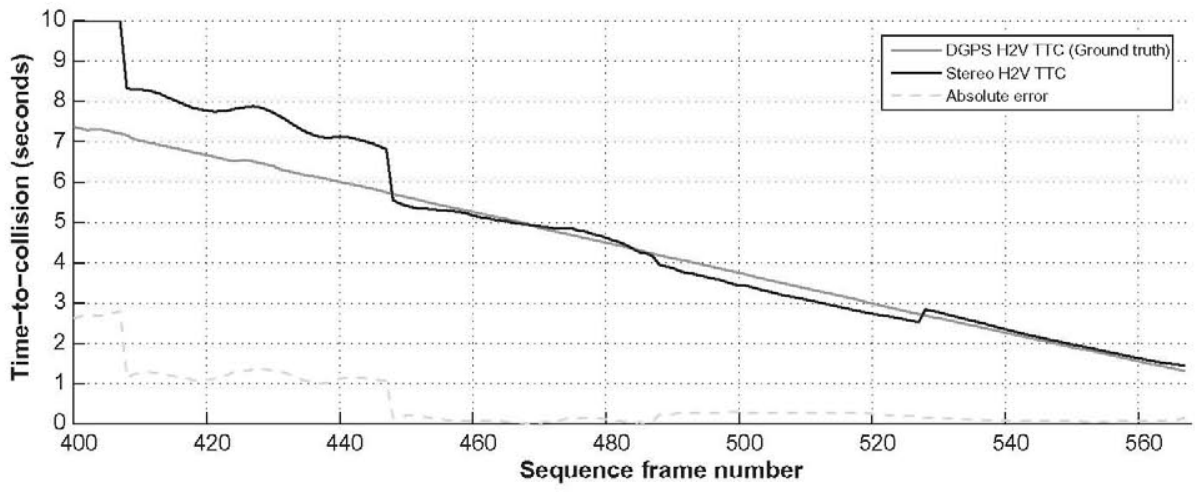

Fig. 11. Stereo vision and DGPS H2V TTC and absolute error in the experiment overtaking the car.

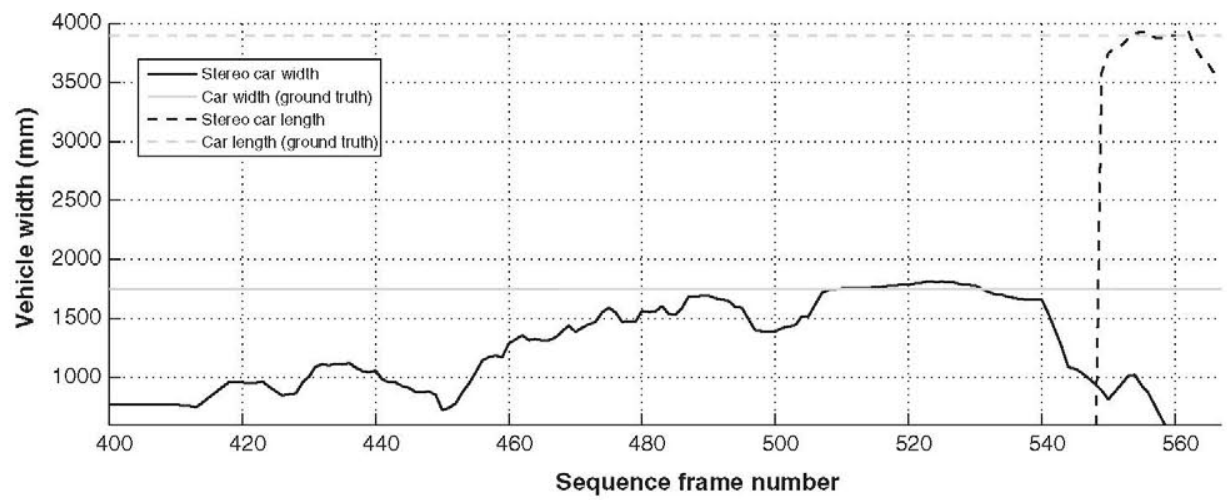

Fig. 12. Stereo video estimate of the car's width and length, and ground truth.

Table 1

RMSE of the relative speed estimate, and range.

\begin{tabular}{llll}
\hline Type of vehicle & CAN speed $(\mathrm{km} / \mathrm{h})($ ground truth) & Speed RMSE $(\mathrm{km} / \mathrm{h})$ & Range $(\mathrm{m})$ \\
\hline Motorbike & 21.2981 & 2.9943 & 34 \\
Car & 22.8 & 3.2075 & 44 \\
Truck & 42.4734 & 3.1717 & 45 \\
\hline
\end{tabular}

$3 \mathrm{~km} / \mathrm{h}$. The mean relative depth error is below $3 \%$ in all cases. The mean relative TTC error is about $3 \%$ for both the car and the truck, and some $6 \%$ for the motorbike for a TTC of less than $6 \mathrm{~s}$. Vehicle width estimates at long distances are unreliable, but for distances less than $30 \mathrm{~m}$ the width RMSE allows a safety lateral offset to be defined for the overtaking manœuvre depending on the type of leading vehicle. With respect to the vehicle length estimates, firstly, if the leading vehicle is a motorbike, length detection is 
Table 2

RMSE of the H2V depth estimate.

\begin{tabular}{|c|c|c|c|c|}
\hline Type of vehicle & Depth RMSE (m) & Depth RMSE (m) depth $<30 \mathrm{~m}$ & Depth RMSE (m) depth $<20 \mathrm{~m}$ & Relative depth error mean (\%) \\
\hline Motorbike & 0.7086 & 0.2932 & 0.1690 & 1.62 \\
\hline Car & 0.9041 & 0.3678 & 0.1490 & 1.97 \\
\hline Truck & 1.7443 & 0.2458 & 0.2100 & 2.91 \\
\hline
\end{tabular}

Table 3

RMSE of the H2V TTC estimate.

\begin{tabular}{|c|c|c|c|c|}
\hline Type of vehicle & TTC RMSE (s) & TTC RMSE $(s)($ TTC $<6 s)$ & TTC RMSE $(s)($ TTC $<3 s)$ & Relative TTC error mean $(\%)($ TTC $<6 \mathrm{~s}$ ) \\
\hline Motorbike & 1.2966 & 0.3790 & 0.2116 & 6.29 \\
\hline Car & 0.9314 & 0.1782 & 0.1538 & 2.9 \\
\hline Truck & 2.2386 & 0.1883 & 0.1056 & 3.1 \\
\hline
\end{tabular}

Table 4

RMSE of the width estimate.

\begin{tabular}{|c|c|c|c|c|}
\hline Type of vehicle & Width (m) (ground truth) & Width RMSE (m) & Width RMSE $(\mathrm{m})$ depth $<30 \mathrm{~m}$ & Width RMSE $(\mathrm{m})$ depth $<20 \mathrm{~m}$ \\
\hline Motorbike & 0.74 & 0.1577 & 0.1021 & 0.0403 \\
\hline Car & 1.75 & 0.5860 & 0.1514 & 0.0541 \\
\hline Truck & 2.8 & 0.6308 & 0.2396 & 0.0202 \\
\hline
\end{tabular}

Table 5

RMSE of the length estimate.

\begin{tabular}{lll}
\hline Type of vehicle & Length $(\mathrm{m})$ (ground truth) & Length RMSE $(\mathrm{m})$ \\
\hline Motorbike & 2.8 & - \\
Car & 3.9 & 0.1744 \\
Truck & 14 & 1.3710 \\
\hline
\end{tabular}

impossible because the measurements are too noisy. Accordingly, if the vehicle ahead is detected to be a motorbike (using the estimated width), a fixed length is defined in order to ensure sufficient space for overtaking. And secondly, for the car and the truck, the estimated respective length RMSEs were $0.1744 \mathrm{~m}$ and $1.3710 \mathrm{~m}$, representing relative errors of about $5 \%$ and $10 \%$, respectively. In order to ensure a safety margin when providing the leading vehicle's length to the fuzzy controller, we increase the final value of the estimated length by $20 \%$.

\section{Control system}

This section describes the lateral and longitudinal controllers, with an explanation of how and when to switch to the overtaking controllers. The control is performed using fuzzy logic.

\subsection{Controller description}

Prior to proceeding with the design of the controller, we established the prerequisites constraining an overtaking manœuvre. Spain's Road Circulation Code imposes two constraints: (a) the time to carry out the overtaking manœuvre must be less than $15 \mathrm{~s}$; (b) the overtaken vehicle cannot increase its speed once the manœuvre has been triggered.

It was also necessary to consider the phases into which overtaking can be divided. It is widely accepted that the manœuvre can be treated as consisting of three phases (Naranjo et al., 2008; Pérez, Milanés, Alonso, Onieva, \& de Pedro, 2010; Shamir, 2004): first, from the time when overtaking is initiated to the time at which the overtaker's nose is parallel with the overtaken's rear-end, i.e., the phase of changing lanes in order to overtake; second, up to the time when the overtaker's rear-end is parallel with the over- taken's nose, i.e., the phase during which the overtaker is driving in the overtaking lane; and third, up to the time at which the overtaker has returned to the original, non-overtaking lane.

The control design is aimed at performing overtaking as humans do, subject to the aforementioned two constraints. Two fuzzy controllers were developed, one for the longitudinal and one for the lateral control.

\subsubsection{Longitudinal controller}

The manœuvre is triggered via the stereo vision system when the TTC is less than $6 \mathrm{~s}\left(t_{0}\right)$. This value was selected based on the accuracy results obtained with the vision-based detection system (see Section 4.2). When overtaking is initiated, the overtaker's speed is already greater than the overtaken's speed, but the driver accelerates further in order to complete the manœuvre as soon as possible.

Using relative velocities $V_{r}$ and distances $d_{r}$, the time intervals $t_{\phi}$ of each phase can be written in general as

$t_{\phi}=\frac{d_{r}}{V_{r}}=\frac{d_{r}}{V_{2}-V_{1}}$

where $V_{2}$ and $V_{1}$ are the mean overtaker's and overtaken's speeds in each phase.

For simplicity, in the following the overtaken's speed $V_{1}$ will be considered constant. The incremental equation of (1) in phase 1 , where $d_{r}=D$ is the distance between the two vehicles at the beginning of the manœuvre, yields

$t_{\phi 1}=\frac{\Delta d_{r}}{\Delta V_{r}}=\frac{D}{\left(V_{2}\left(t_{\phi 1}\right)-V_{1}\left(t_{\phi 1}\right)\right)-\left(V_{2}\left(t_{0}\right)-V_{1}\left(t_{0}\right)\right)}$

It is then straightforward to see that the overtaker's speed at the end of the first phase would be

$V_{2}\left(t_{\phi 1}\right)=V_{2}\left(t_{0}\right)+\frac{D}{t_{\phi 1}}$

This value is limited by the maximum allowed speed.

The reference speed in the second phase is also obtained using Eq. (1)

$V_{2}(t)=V_{1}+\frac{L_{1}+\alpha L_{2}}{t_{\phi 2}}, \quad \forall t \in\left[t_{\phi 1}, t_{\phi 2}\right]$ 
where $L_{2}$ is set to $3.6 \mathrm{~m}$ and $L_{1}$ is obtained from the stereo vision system $-\mathrm{a} \alpha=1.2$ scale factor is applied to take associated errors into account - once the two vehicles are in different lanes. This value is compared with $V_{2}\left(t_{\phi 1}\right)$ and the greater is used as the reference speed for this phase.

Since the maximum allowable time to perform overtaking is $15 \mathrm{~s}$ and several factors can lead to major delays in its execution, an overall $8 \mathrm{~s}$ interval was set for our experimental trials. Following the analytical results of Shamir (2004), the time to perform the first and third phases was set to $t_{\phi 1}=3 \mathrm{~s}$. The time for the second phase was limited to $t_{\phi 2}=2 \mathrm{~s}$. Finally, the third phase will be similar to the first, but will be finished faster $\left(t_{\phi 3} \leqslant 3 \mathrm{~s}\right)$.

The speed controller designed to follow these reference speeds is based on a previously developed controller with some modifications (Onieva et al., 2010) to permit maximum comfort in acceleration. Fig. 13 depicts the control surface of the fuzzy longitudinal controller. As input values, the actual Velocity and Velocity Error were used. The output was the Pedal variable defined in the interval $[-1,1]$. Values from -1 to 0 represent braking actions, and values from 0 to 1 throttle (acceleration) actions. The slower the actual speed, the smaller the throttle action, the goal being to avoid undesired sharp accelerations - greater than $2 \mathrm{~m} / \mathrm{s}^{2}$.

\subsubsection{Lateral controller}

The obstacle avoidance literature includes several proposals of procedures to estimate the optimal trajectory to take in such demanding situations, and to decide whether it is more convenient to brake than to steer. One proposal (Horiuchi, Okada, \& Nohtomi, 2004) is to use, under reasonable assumptions, the following formula for the maximum speed in steering to avoid an obstacle:

$V_{\max } \leqslant D \sqrt{\frac{\mu g}{2 H}}$

where $V_{\max }$ is the maximum speed a vehicle can move at to avoid an obstacle located $D$ meters ahead by obtaining a lateral displacement of $\mathrm{H}$ meters. This inequality assumes that the manœuvre involves the maximum lateral tyre potential, $\gamma_{\max }=\mu \mathrm{g}$, where $\mu$ is the tyre-road coefficient of friction.

If $V_{\max }$ is replaced by the relative velocity $V_{r}$, and a comfortable lateral acceleration $\left(\gamma_{c}=1 \mathrm{~m} / \mathrm{s}^{2}\right.$, (BECHTEL, 1993)) is used instead of the maximum lateral acceleration $\gamma_{\max }$, then Eq. (5) can be rewritten to obtain the maximum lateral displacement that ensures a comfortable manœuvre

$H=\frac{\gamma_{c} D^{2}}{2 V_{r}^{2}}$

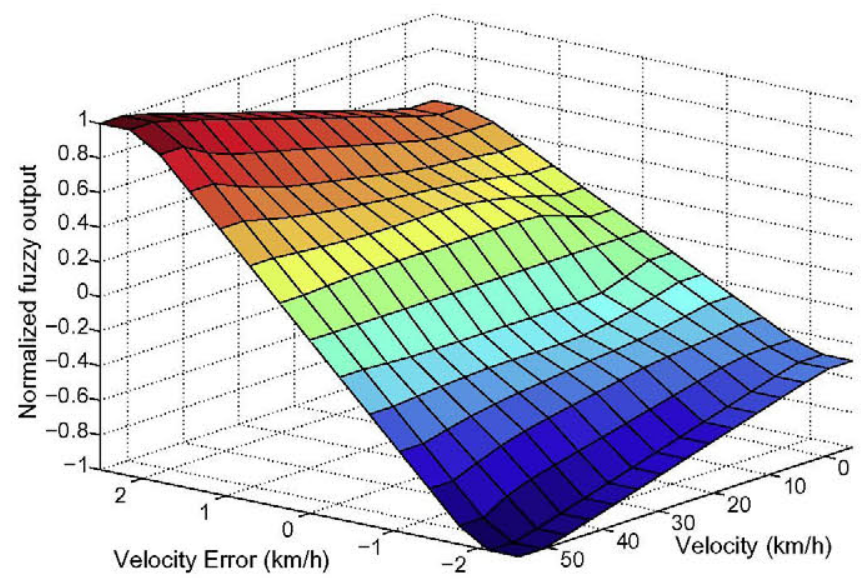

Fig. 13. Throttle and brake control surface. where $L$ and $V_{r}$ will be provided by the stereo vision system. Note from Fig. 1 that the final lateral displacement $H$ should be less than the distance to the left (far) edge of the road $H_{l}$ and greater than the lateral distance between the vehicles:

$d_{s}+\frac{P V_{W}}{2}+\frac{O V_{W}}{2} \leqslant H \leqslant H_{l}$

where $d_{s}$ is a safe distance that will be set at $1 \mathrm{~m}$, and $O V_{W}$ is the host vehicle's own width. The maximum lateral displacement is determined by forcing compliance with the proposed times for the overtaking manœuvre $-3 \mathrm{~s}$ for the lane-change. If $T T C=3 \mathrm{~s}$, the distance between the two vehicles is $D=3 V_{r}$. Hence, from Eq. (6), $H=4.5 \mathrm{~m}$. Assuming lanes of $3 \mathrm{~m}$ in width, the maximum lateral displacement during the manœuvre has to be less than or equal to $H_{l}=4.5 \mathrm{~m}$. Hence the time allowed for the lane change is consistent with the problem's constraints.

A fuzzy-logic based system was developed to perform the lateral control. Two variables were considered to implement the steering wheel management:

Velocity whose function is to maintain the comfort of the manœuvre - the greater the speed, the lighter the action on the steering wheel and vice versa. The definition of its membership function is shown in the top plot of Fig. 14.

Lateral Displacement whose function is to determine how many meters the overtaker has to move laterally so as to satisfy the 1.5 meter lateral displacement requirement. The goal is to perform not a lane change but a lateral displacement depending on the vehicle to be overtaken. The definition of its membership function is shown in the middle plot of Fig. 14.

The output is the steering wheel position normalized to the interval $[-1,1]$ where -1 indicates the maximum displacement to the left and 1 the maximum displacement to the right. Seven singleton-type membership functions were defined to codify the output variables (see bottom plot of Fig. 14). Fig. 15 shows the control surface for the fuzzy lateral controller.

\subsection{Controller validation}

Before including the vision-based system to detect the preceding vehicle, the lateral and longitudinal controllers were both validated. To this end, a GPS point was located in the center of the right lane of one of the straight stretches at the CAR's private driving circuit. This point was taken to be the rear-end of a motionless preceding vehicle. The distance between vehicles was dynamically calculated using the DPGS/IMU positioning system. The width and the length were then introduced via software so as to check the controllers. Table 6 lists the values used as reference.

Fig. 16 shows the results using different vehicles. The upper plot depicts the evolution of the autonomous system in $X-Y$ coordinates. The lower plot represents the speed of the vehicle during the manœuvre. One can appreciate the instants at which the manœuvre is triggered $-T T C=6 \mathrm{~s}$. From that moment, the reference speed is set to a higher value so as to ensure that the manœuvre is completed in time. $T T C=0 \mathrm{~s}$ indicates the instant when the overtaker's nose is parallel with the overtaken's rear-end. At that moment, the minimum lateral displacement requirement has to be satisfied. In all the cases, the lateral displacement was greater than the minimum required displacement.

Note the difference in trajectories depending on the type of the preceding vehicle. In the case of a motorbike, the two vehicles are parallel before the overtaker has left the right lane but is maintaining a lateral safety distance as human drivers do. The steering change is mainly done during the first instants of the manœuvre. 

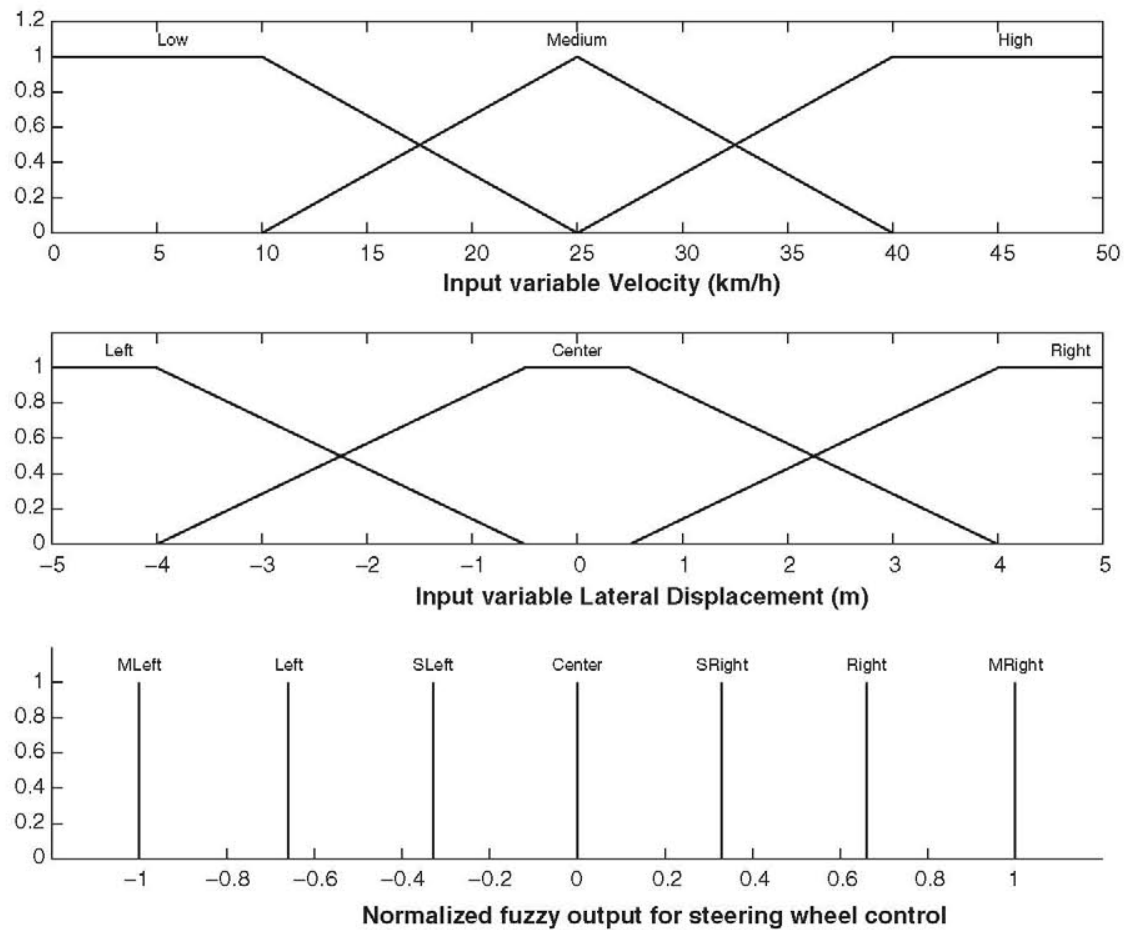

Fig. 14. The input and output variables of the steering wheel controller for automatic overtaking manœuvres.

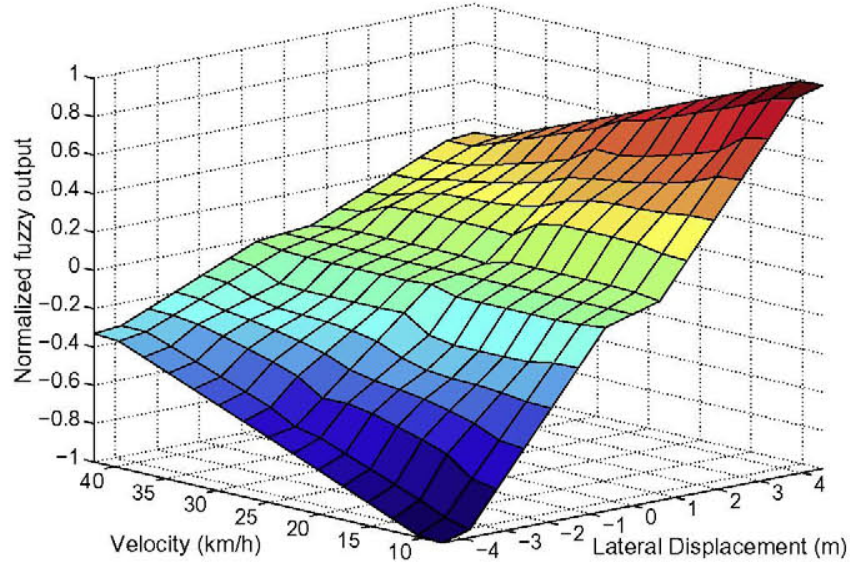

Fig. 15. Steering wheel control surface.

Table 6

Lengths and widths for the leading vehicle.

\begin{tabular}{lll}
\hline Type of vehicle & Length $(\mathrm{m})$ & Width $(\mathrm{m})$ \\
\hline Motorbike & 1 & - \\
Car & 4 & 1.5 \\
Truck & 12 & 2.5 \\
\hline
\end{tabular}

The wider the preceding vehicle, the greater is the lateral displacement.

With respect to the speed, in the trial in which the vehicle to be overtaken is a truck, there is a further change in the reference speed when the two vehicles are parallel. Once the manœuvre has been completed, the reference speed is reset to its initial value. All the trials satisfy the initial requirements, demonstrating that the controllers developed are capable of performing autonomous overtaking with adequate safety.
Table 7 summarizes the lateral displacement errors at the beginning of the second phase - the overtaker's nose is in parallel with overtaken's rear-end - corresponding to the three vehicles.

\section{System integration}

The results of an experiment with the integration of the two fuzzy controllers and the stereo vision system are shown in Fig. 17. The systems were mounted in the prototype vehicle (Fig. 2). The overtaken vehicle was an electric Citroën Berlingo van which has been used in numerous experiments in the AUTOPIA program (Milanés, Pérez, Onieva, González, \& de edro, 2010; Naranjo et al., 2005, 2008; Pérez \& Milanés et al., 2010). It was equipped with a DGPS receiver and a differential Hall effect sensor coupled to a cogwheel attached to one of the forward wheels (Pérez et al., 2010b) so as to log its position and speed, respectively.

For the trial, a straight stretch of road of 200 meters in CAR's facilities was used. The overtaken vehicle is started and when it reaches a reference speed close to $8 \mathrm{~km} / \mathrm{h}$, the overtaking vehicle is started. As can be seen, the overtaking vehicle's reference speed is set at $15 \mathrm{~km} / \mathrm{h}$ so as to mimic a real traffic situation in which the overtaking vehicle detects a slower preceding vehicle.

The vision-based system is continuously checking the vicinity of the host vehicle. When the host vehicle is sufficiently close to the preceding one (TTC=6s), the first phase of the overtaking manœuvre is initiated. To ensure a rapid manœuvre, the target speed is raised to $26.6 \mathrm{~km} / \mathrm{h}$ according to Eq. (3). Note also that at the end of this phase the overtaking vehicle is already in the opposite lane, and the two vehicles are separated by more than $2 \mathrm{~m}$ laterally. The second phase is then enabled until the overtaking vehicle is able to return to its original lane. Finally, the overtaking vehicle performs the third phase of the manœuvre, returning to the right-hand lane. Once the manœuvre has been completed, the reference speed is reset to the initial value, and the host vehicle continues on its way. 

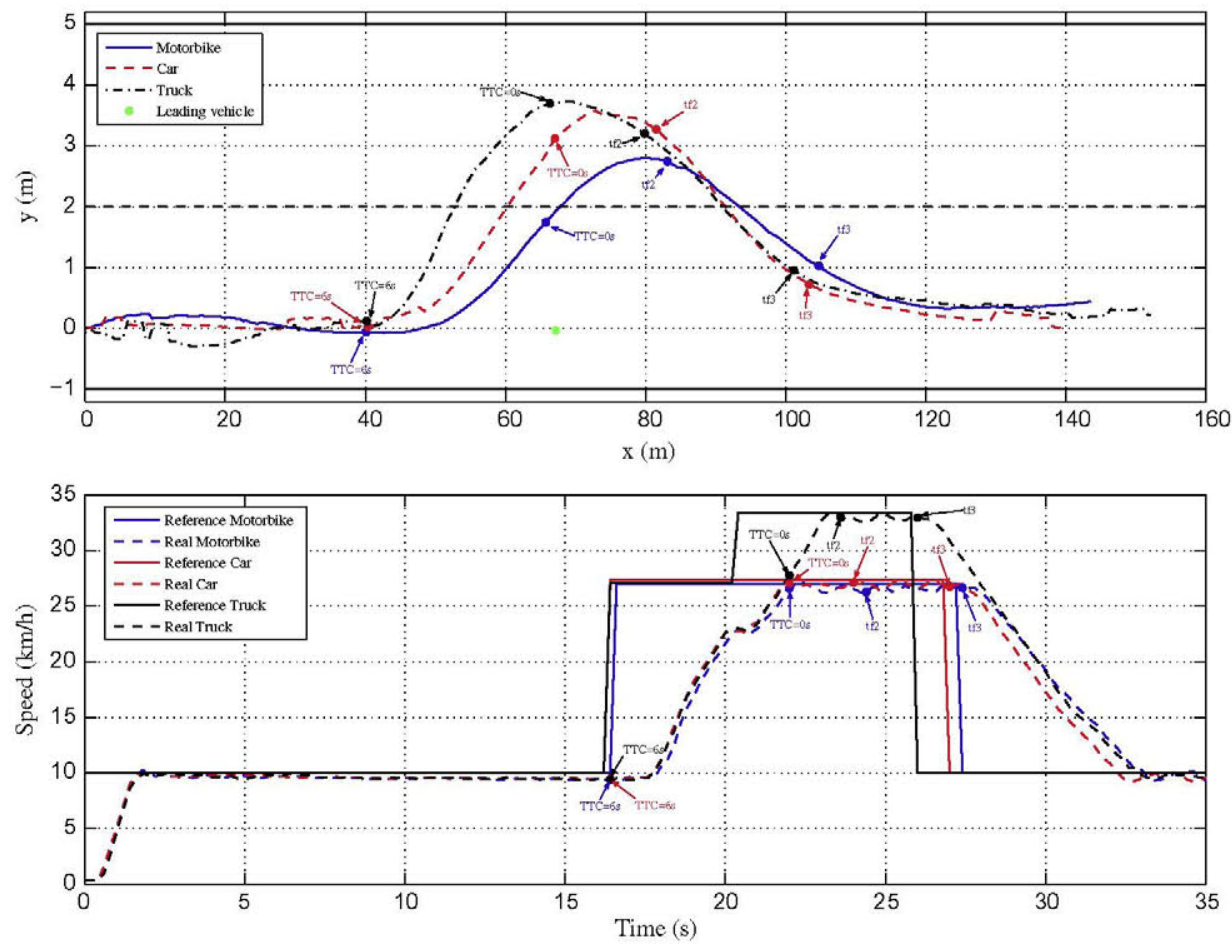

Fig. 16. Test results for validation of the lateral controller with motorbike, car, and truck.

Table 7

Distance between vehicles at TTC $=0$.

\begin{tabular}{lll}
\hline Type of vehicle & Distance mean $(\mathrm{m})$ & Distance RMSE $(\mathrm{m})$ \\
\hline Motorbike & 1.87 & 0.12 \\
Car & 2.76 & 0.13 \\
Truck & 3.36 & 0.15 \\
\hline
\end{tabular}

\section{Concluding remarks and next steps}

Autonomous systems to aid the driver in specific tasks are rapidly changing from being a utopian dream to becoming an everyday reality. The present communication has presented our approach towards solving one of the major causes of fatalities in
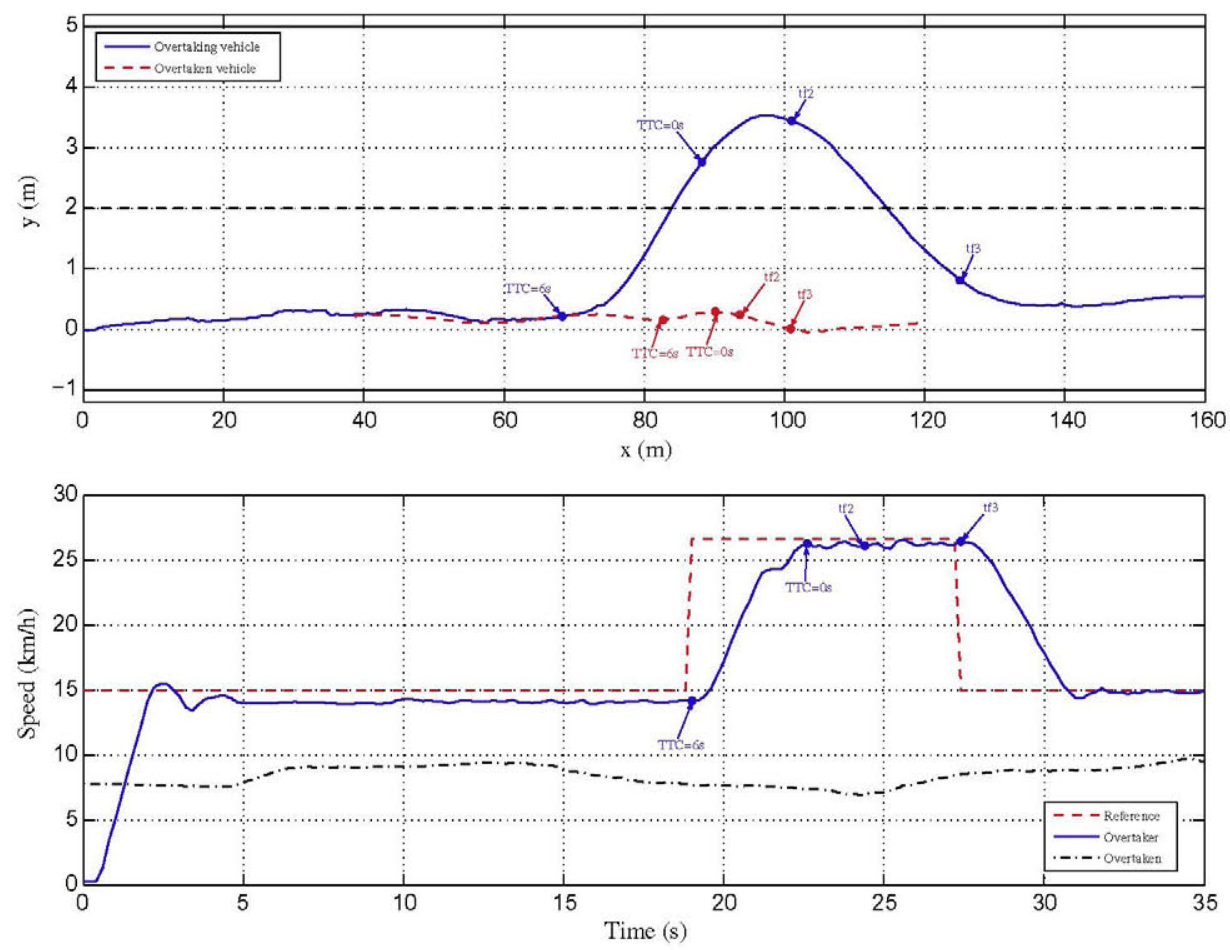

Fig. 17. Overtaking experiment using a moving car as the overtaken vehicle. 
road transport: overtaking. The proposal is based on a stereo vision system to detect the preceding vehicle and then to activate the autonomous overtaking system. A commercial car was instrumented to manage its actuators autonomously. The system was tested in a real environment with good results.

The vision system was capable of detecting the preceding vehicle and its speed with sufficient accuracy, distinguishing between motorbikes, cars, and trucks. Once the width of the preceding vehicle had been detected and the autonomous manouvre activated, the system was capable of determining the length of the preceding vehicle to modify the host's speed so as to reduce the time it spends in the overtaking lane.

The longitudinal controller was a modification of a previously developed controller (Onieva et al., 2010) aimed at achieving the maximum comfortable acceleration $\left(2 \mathrm{~m} / \mathrm{s}^{2}\right)$ during the manœuvre so as to reduce its duration while maintaining a smooth ride for the occupants.

The lateral controller was a newly developed fuzzy controller designed to perform the steering change autonomously. As inputs, the controller uses signals from the vision system - lateral displacement and speed - and a DGPS/IMU system to obtain the vehicle's positioning with sufficient accuracy.

The system was designed to satisfy constraints on overtaking time and safety in the lateral displacement. The wider the preceding vehicle the greater the lateral displacement has to be, and the longer the preceding vehicle the greater the speed. The experimental results demonstrated the proposed system to be capable of providing a good solution to the automation of this manœuvre.

While the results achieved are promising, there still remain several questions in need of further investigation. Among them, the detection of oncoming traffic via stereo vision, increasing the vehicle detection range, and performing the autonomous lateral displacement at greater speeds will be studied as part of future research. To this end, the fuzzy controllers will have to be modified to take these new scenarios into account.

\section{Acknowledgements}

This work has been supported by the Spanish Ministry of Science and Innovation by means of Research Grant TRANSITO TRA2008-06602 and Spanish Ministry of Development by means of Research Grant GUIADE P9/08.

\section{References}

BECHTEL (1993). Compendium of executive summaries from the maglev system concept definition final reports. Technical Report. US Department of Transportation.

Broggi, A., Bertozzi, M., Fascioli, A., Lo, C., \& Piazzi, B. (1999). The argo autonomous vehicle's vision and control systems. International Journal of Intelligent Control Systems, 3, 409-441.

Castro, J., Delgado, M. J., \& Medina, M. R. L. (2011). An expert fuzzy system for predicting object collisions. its application for avoiding pedestrian accidents. Expert Systems with Applications, 38, 486494.

Chang, B., Tsai, H., \& Young, C. (2010). Intelligent data fusion system for predicting vehicle collision warning using vision/gps sensing. Expert Systems with Applications, 37, 2439-2450.

Chellappa, R., Qian, G., \& Zheng, Q. (2004). Vehicle detection and tracking using acoustic and video sensors. In Proceedings IEEE international confernce on acoustics, speech, and signal processing (ICASSP'04).

Feng, Y., Rongben, W., \& Ronghui, Z. (2008). Research on lane changing and overtaking for intelligent vehicle based on vision navigation. In Proceedings of the IEEE international conference on industrial technology ICIT 2008 (pp. 1-6).

Geronimo, D., Lopez, A. M., Sappa, A. D., \& Graf, T. (2010). Survey of pedestrian detection for advanced driver assistance systems. IEEE Transactions on Pattern Analysis and Machine Intelligence, 32, 1239-1258.

Godbole, D., \& Lygeros, J. (1993). Longitudinal control of the lead car of a platoon. Technical Report. California Partners for Advanced Transit and Highways.

Hancock, J., Langer, D., Hebert, M., Sullivan, R., Ingimarson, D., Hoffman, E. Mettenleiter, \& M., Froehlich, C. (1998). Active laser radar for highperformance measurements. In Proceedings of the IEEE international robotics and automation conference (pp. 1465-1470).
Horiuchi, S., Okada, K., \& Nohtomi, S. (2004). Optimum steering and braking control strategies in obstacle avoiding maneuvers. In 7 th international symposium on advanced vehicle control AVECO4 (pp. 619-624).

Kestinga, A., Treibera, M., Schönhofa, M., \& Helbing, D. (2008). Adaptive cruise control design for active congestion avoidance. Transportation Research Part $C$ Emerging Technologies, 16, 668-683.

Lindgren, A., Angelelli, A., Mendoza, P. A., \& Chen, F. (2009). Driver behaviour when using an integrated advisory warning display for advanced driver assistance systems. IET Intelligent Transport Systems, 3, 390-399.

Llorca, D. F., Sánchez, S., Ocaña, M., \& Sotelo, M. A. (2010). Vision-based traffic data collection sensor for automotive applications. Sensors, 10, 860-875.

Llorca, D. F., Sotelo, M. A., Parra, I., Naranjo, J. E., Gavilan, M., \& Alvarez, S. (2009). An experimental study on pitch compensation in pedestrian-protection systems for collision avoidance and mitigation. IEEE Transactions on Intelligent Transportation Systems, 10, 469-474.

Llorca, D. F., Sotelo, M. A., Parra, l., Ocaña, M. \& Bergasa, L. M. (2010). Error analysis in a stereo vision-based pedestrian detection sensor for collision avoidance applications. Sensors, 10, 3741-3758.

Lu, M., Wevers, K., van der Heijden, R., \& Heijer, T. (2004). Adas applications for improving traffic safety. In Proceedings of the IEEE International Systems, Man and Cybernetics Conference (pp. 3995-4002)

Mandellos, N. A., Keramitsoglou, I., \& Kiranoudis, C. (2011). A background subtraction algorithm for detecting and tracking vehicles. Expert Systems with Applications, 38, 1619-1631.

Milanés, V., González, C., Naranjo, J., Onieva, E., \& De Pedro, T. (2010). Electrohydraulic braking system for autonomous vehicles. International Journal of Automotive Technology, 11, 89-95.

Milanés, V., Naranjo, J. E., Gonzalez, C., Alonso, J., \& de Pedro, T. (2008). Autonomous vehicle based in cooperative gps and inertial systems. Robotica, 26, 627-633.

Milanés, V., Pérez, J., Onieva, E., Gonzâlez, C., \& de edro, T. (2010). Lateral power controller for unmanned vehicles. Przeglad Elektrotechniczny, Electrical Review $86,207-211$.

Naranjo, J. E., Gonzalez, C. Garcia, R., \& de Pedro, T. (2008). Lane-change fuzzy control in autonomous vehicles for the overtaking maneuver. IEEE Transactions on Intelligent Transportation Systems, 9, 438-450.

Naranjo, J. E., Gonzalez, C., Garcia, R., de Pedro, T., \& Haber, R. E. (2005). Powersteering control architecture for automatic driving. IEEE Transactions on Intelligent Transportation Systems, 6, 406-415.

Onieva, E., Milanés, V., González, C., de Pedro, T., Pérez, J., \& Alonso, J. (2010) Throttle and brake pedals automation for populated areas. Robotica, 28, 509-516.

Park, S. J., Kim, T. Y., Kang, S. M., \& Koo, K. H. (2003). A novel signal processing technique for vehicle detection radar. In Proceedings on IEEE MTT-S International Microwave Symposium Digest (pp. 607-610).

Parra, I., Llorca, D. F., Sotelo, M. A., Bergasa, L. M., de Toro, P. R., Nuevo, J., et al. (2007). Combination of feature extraction methods for SVM pedestrian detection. IEEE Transactions on Intelligent Transportation Systems, 8, 292-307.

Peng, Y. (2010). Adaptive intelligent backstepping longitudinal control of vehicle platoons using output recurrent cerebellar model articulation controller. Expert Systems with Applications, 37, 20162027.

Perez, A., Gatcia, M. I., Nieto, M., Pedraza, J. L., Rodriguez, S., \& Zamorano, J. (2010) Argos: An advanced in-vehicle data recorder on a massively sensorized vehicle for car driver behavior experimentation. IEEE Transactions on Intelligent Transportation Systems, 11, 463-473.

Pérez, J., Milanés, V., Alonso, J., Onieva, E., \& de Pedro, T. (2010). Adelantamiento con vehiculos autónomos en carreteras de doble sentido. Revista Iberoamericana de Automática e Informática Industrial, 7, 25-33.

Pérez, J., Seco, F., Milanés, V., Jiménez, A., Dfaz, J., \& de Pedro, T. (2010b). An rfidbased intelligent vehicle speed controller using active traffic signals. Sensors, 10 , $5872-5887$.

Pomerleau, D. (1995). Ralph: Rapidly adapting lateral position handler. In Proceedings of the Intelligent Vehicles'95 Symposium (pp. 506-511)

Sun, Z., Bebis, G., Miller, R., 2006. On-road vehicle detection: a review. IEEE Transactions on Pattern Analysis and Machine Intelligence 28, 694-711.

Shamir, T. (2004). How should an autonomous vehicle overtake a slower moving vehicle: design and analysis of an optimal trajectory. IEEE Transactions on Automatic Control, 49, 607-610.

Stein, G. P., Mano, O., \& Shashua, A. (2003). Vision-based acc with a single camera: bounds on range and range rate accuracy. In Proceedings of the IEEE intelligent vehicles symposium (pp. 120-125)

Usman, G., \& Kunwar, F. (2009). Autonomous vehicle overtaking - an online solution. In Proceedings of the IEEE International Conference on Automation and Logistics ICAL'09 (pp. 596-601).

Wang, J., Bebis, G., \& Miller, R. (2005). Overtaking vehicle detection using dynamic and quasi-static background modeling. In Proceedings of the CVPR workshops computer vision and pattern recognition - Workshops IEEE computer society conference (p. 64)

Wang, C. C., Thorpe, C., \& Suppe, A. (2003). Ladar-based detection and tracking of moving objects from a ground vehicle at high speeds. In Proceedings of the IEEE intelligent vehicles symposium (pp. 416-421)

Wang, J., Lin, C., \& Chen, S. (2010). Applying fuzzy method to vision-based lane detection and departure warning system. Expert Systems with Applications, 37, 113126.

Wang, J., Schroedl, S., Mezger, K., Ortloff, R., Joos, A., \& Passegger, T. (2005). lane keeping based on location technology. IEEE Transactions on Intelligen Transportation Systems, 6, 351-356. 
Wang, F., Yang, M., \& Yang, R. (2009). Conflict-probability-estimation-based overtaking for intelligent vehicles. IEEE Transactions on Intelligent Transportation Systems, 10, 366-370.

Wu, S. J., Chiang, H. H., Perng, J. W., Chen, C. J., Wu, B. F., \& Lee, T. T. (2008). The heterogeneous systems integration design and implementation for lane keeping on a vehicle. IEEE Transactions on Intelligent Transportation Systems, 9, 246-263.
Zhu, Y., Comaniciu, D., Pellkofer, M., \& Koehler, T. (2006). Reliable detection of overtaking vehicles using robust information fusion. IEEE Transactions on Intelligent Transportation Systems, 7, 401-414. 